\title{
BMJ Open Rationale, design and study protocol of the randomised controlled trial: Diabetes Interventional Assessment of Slimming or Training tO Lessen Inconspicuous Cardiovascular Dysfunction (the DIASTOLIC study)
}

Gaurav Singh Gulsin, ${ }^{1}$ Emer M Brady, ${ }^{2}$ Daniel J Swarbrick, ${ }^{1}$ Lavanya Athithan, ${ }^{1}$ Joseph Henson, ${ }^{3}$ Emma Baldry, ${ }^{2}$ John McAdam, ${ }^{1}$ Anna-Marie Marsh, ${ }^{1}$ Kelly S Parke, ${ }^{1}$ Joanne V Wormleighton, ${ }^{1}$ Eylem Levelt, ${ }^{4}$ Thomas Yates, ${ }^{2,3}$ Danielle Bodicoat, ${ }^{2}$ Kamlesh Khunti, ${ }^{2}$ Melanie J Davies, ${ }^{2}$ Gerry P McCann ${ }^{1}$

To cite: Gulsin GS, Brady EM, Swarbrick DJ, et al. Rationale, design and study protocol of the randomised controlled trial: Diabetes Interventional Assessment of Slimming or Training to Lessen Inconspicuous Cardiovascular Dysfunction (the DIASTOLIC study). BMJ Open 2019;9:e023207. doi:10.1136/ bmjopen-2018-023207

- Prepublication history for this paper is available online. To view these files, please visit the journal online (http://dx.doi org/10.1136/bmjopen-2018023207).

Received 27 March 2018 Revised 10 January 2019 Accepted 5 February 2019

Check for updates

(C) Author(s) (or their employer(s)) 2019. Re-use permitted under CC BY-NC. No commercial re-use. See rights and permissions. Published by BMJ.

For numbered affiliations see end of article.

Correspondence to Dr Gerry P McCann; gpm12@leicester.ac.uk

\section{ABSTRACT}

Introduction Despite their young age and relatively short duration of disease, younger adults with type 2 diabetes (T2D) already have diastolic dysfunction and may be at risk of incipient heart failure. Whether weight loss or exercise training improve cardiac dysfunction in people with $\mathrm{T} 2 \mathrm{D}$ remains to be established.

Methods and analysis Prospective, randomised, openlabel, blind endpoint trial. The primary aim of the study is to determine if diastolic function can be improved by either a meal replacement plan or a supervised exercise programme, compared with guideline-directed care. A total of 90 obese participants with T2D (aged 18-65 years), diabetes duration $<12$ years and not on insulin treatment will be randomised to either guideline-directed clinical care with lifestyle coaching, a low-energy meal replacement diet (average $\approx 810 \mathrm{kcal} /$ day) or a supervised exercise programme for 12 weeks. Participants undergo glycometabolic profiling, cardiopulmonary exercise testing, echocardiography and MRI scanning to assesses cardiac structure and function and dual-energy X-ray absorptiometry scanning for body composition. Key secondary aims are to assess the effects of the interventions on glycaemic control and insulin resistance, exercise capacity, blood pressure, changes in body composition and association of favourable cardiac remodelling with improvements in weight loss, exercise capacity and glycometabolic control.

Ethics and dissemination The study has full ethical approval, and data collection was completed in August 2018. The study results will be submitted for publication within 6 months of completion.

Trial registration number NCT02590822; Pre-results.

\section{BACKGROUND}

Recent estimates suggest there are currently 425 million people with type 2 diabetes mellitus (T2D) globally. ${ }^{1}$ The dramatic rise
Strengths and limitations of this study

- There are few published studies demonstrating the beneficial effects of weight loss or exercise programmes on cardiac remodelling and function in people with type 2 diabetes (T2D).

- The Diabetes Interventional Assessment of Slimming or Training to Lessen Inconspicuous Cardiovascular Dysfunction study will comprehensively describe changes in cardiac structure and function in younger obese people with T2D treated with either a low-energy meal replacement diet or exercise programme.

- This is a proof-of-efficacy study, and therefore, effects on clinical outcomes is not planned with a short duration of follow-up.

- Only patients who are able to exercise and willing to undertake the meal replacement plan are eligible, and we have limited our inclusion to younger adults ( $<65$ years) with a maximum duration of diabetes of 12 years, so the results will not necessarily be generalisable to all people with T2D.

Recruitment to the study ended in August 2018.

in the levels of obesity and sedentary lifestyles in younger age groups has resulted in up to a 10-fold increase in the prevalence of T2D in younger adults. ${ }^{2}$ Whereas T2D was once a rarity in young people, increasingly we are seeing the condition diagnosed in children, adolescents and adults under the age of 30 years. ${ }^{34}$ The onset of T2D in younger people represents an extreme phenotype that magnifies the disease profile observed in older adults. This is of clinical significance and represents an important public health issue. 
The most deleterious consequence of developing T2D is a substantially elevated risk of cardiovascular disease (CVD), a feature that is magnified in younger adults. For example, the 20-year mortality rate in younger adults with T2D is as high as $11 \%$, the majority from CVD. ${ }^{5}$ Furthermore, $48 \%$ of young adults with obesity at 22 years of age experience some form of CVD or early mortality with a life expectancy of 55 years. ${ }^{6}$ This is a rapidly emerging clinical pattern, where the mechanisms driving the high disease burden in younger adults with T2D, and potential therapeutic strategies, have not been fully elucidated.

While the risk of developing coronary artery disease and stroke are well recognised, the association of diabetes with heart failure (HF) has received less attention. ${ }^{7}$ Diabetes is an independent risk factor for the development of $\mathrm{HF}^{8-10}$ In the 2015-2016 UK National Diabetes Audit, there were 115695 emergency admissions for patients with diabetes and HF (32.5\% of all HF admissions) despite people with diabetes representing only $8 \%$ of the population. Importantly, as many as half of all patients in the community with $\mathrm{HF}$ are asymptomatic. ${ }^{11}$ There is a high prevalence of people with diabetes with both common forms of HF: reduced systolic left ventricular (LV) function and HF with preserved ejection fraction (which represents up to $50 \%$ of cases in the community). ${ }^{12}$

\section{Diabetic cardiomyopathy: the precursor to clinical HF}

Diabetic cardiomyopathy is defined as myocardial disease in patients with diabetes, not attributable to hypertension, coronary artery disease or other cardiac disease. ${ }^{13}$ The pathophysiology of diabetic cardiomyopathy is complex, involving metabolic, vascular, inflammatory, epigenetic and neuroendocrine mechanisms. Diastolic (relaxation/ filling) dysfunction and concentric LV remodelling typify most cases, with a prevalence of up to $75 \%$ in adults with T2D. ${ }^{14-16}$ Multiple parameters have been associated with diastolic dysfunction including glycated haemoglobin (HbAlc) level, ${ }^{17}$ age, ${ }^{18}$ aortic stiffness, ${ }^{19}$ duration of diabetes, ${ }^{19}$ microvascular dysfunction ${ }^{20}$ and myocardial triglyceride content. ${ }^{21-23}$

\section{Pilot data: cardiovascular dysfunction in younger adults with T2D}

A pilot Medical Research Council (MRC)-funded study investigating the biochemical and cardiac abnormalities associated with T2D in younger adults (18-40 years) was conducted by our group. ${ }^{19}$ Twenty young adults with diagnosed T2D, 20 age-matched metabolically healthy obese and 10 lean controls were recruited. Cardiac structure and function were assessed by tagged cardiac MRI (CMRI) and myocardial perfusion and scarring were assessed with contrast-enhanced CMRI. There were elevated inflammatory markers (high sensitivity C-reactive protein (hsCRP), interleukin-6 and 10 and tumour necrosis factor-alpha), reduced adiponectin and a strong trend for concentric remodelling (increased LV mass/volume) in both the T2D and obese controls versus the lean controls. The most striking finding was evidence of greater diastolic dysfunction in T2D compared with both the obese and lean controls. ${ }^{19}$ Specifically, peak early diastolic strain rate (PEDSR) had an average value of $1.3 \frac{1}{\mathrm{~s}}$ in the patients with T2D, which was $20 \%$ lower than the obese controls and $30 \%$ lower compared with the lean controls. There was also a strong trend towards reduced systolic strain in T2D $(-21 \%)$ compared with both control groups $(-23 \%$, $\mathrm{p}=0.08)$, which suggests these patients may be at risk of incipient systolic dysfunction and are indeed already at the threshold of early diabetic cardiomyopathy.

These results were consistent with a larger echocardiographic study in adolescents with diabetes compared with lean and obese controls. ${ }^{24}$ There is a growing body of evidence that preclinical diastolic dysfunction is already manifest in younger adults with T2D, despite their relatively young age and short duration of T2D. This is of high clinical relevance and suggests that the high risk of CVD observed in T2D may be linked to diastolic dysfunction, predisposing these patients to $\mathrm{HF}$, in advance of overt systolic compromise. ${ }^{1415}$ Therefore, therapeutic strategies that target both glycaemic control and diastolic cardiac function are highly desirable in younger adults with T2D to prevent the development of HF.

\section{Interventions to reverse diastolic dysfunction in T2D}

Proof that T2D is a reversible condition has been firmly established in patients undergoing bariatric surgery ${ }^{25} 26$ and more recently with intensive primary care-led weight management in the Diabetes Remission Clinical Trial (DiRECT) trial. ${ }^{27}$ The extent of weight loss is strongly linked to reversal of T2D (with a diabetes remission rate of $7 \%$ with weight loss $<5 \mathrm{~kg}$ vs $86 \%$ with weight loss $\geq 15 \mathrm{~kg}),{ }^{27}$ as is good glycaemic control before surgery. ${ }^{26}$ Insulin use, diabetes duration and high HbA1c levels are associated with reduced likelihood of reversal. ${ }^{26}$ These studies, however, have not assessed changes in cardiovascular function.

\section{Low and very low energy diets (VLEDs)}

The phenotypic characteristics of T2D (excess body weight, ectopic fat deposition and insulin resistance) are largely normalised through VLEDs (typically $<800 \mathrm{kcal} /$ day) via a meal replacement approach. Many of the metabolic responses mimic adaptions following bariatric surgery. ${ }^{28}$ A proof-of-concept study showed that just 8 weeks of $510 \mathrm{kcal} /$ day diet via this approach resulted in normalisation of fasting glucose, hepatic glucose production and hepatic triglyceride content with substantial improvements in glucose disposal rates. ${ }^{29}{ }^{30}$ In a larger $(n=298)$ recent trial of a low-energy total diet replacement programme (825-853 kcal/day over $3-5$ months) versus guideline-driven best practice, diabetes remission was achieved in almost half $(46 \%)$ of those who received the diet (mean weight loss $10 \mathrm{~kg}$ ) compared with only $4 \%$ in the comparator group (mean weight loss $1 \mathrm{~kg}$ ). ${ }^{27}$ Many of these improvements are seen within 1 week. The majority of T2D individuals are also found to lose $50 \%$ of excess body weight within 8-12 weeks of VLED. ${ }^{29} 30$ 
In a self-reported study of dietary induced weight loss, diabetes reversal was positively associated with the magnitude of weight loss and inversely with diabetes duration (being lowest in those with diabetes $>8$ years) ${ }^{31}$

In obese subjects without T2D, sustained weight loss, either with diet or after surgery, has resulted in favourable reductions in cardiovascular MRI (CMRI) measured LV mass, volumes, arterial stiffness and diastolic function. ${ }^{32}$ Improved diastolic function following weight loss in obesity has been associated with improved energetics ${ }^{33}$ and with reduced myocardial triglyceride content. ${ }^{34}$ In patients with insulin-treated T2D, a $471 \mathrm{kcal} /$ day VLED has also been shown to reduce myocardial steatosis $(0.88 \% \pm 0.12 \%$ to $0.64 \pm 0.14 \%, \mathrm{p}=0.02)$ in a small $(\mathrm{n}=12)$ single-group study and was associated with improved diastolic filling on CMR. ${ }^{35}$ Interestingly, a brief report from the same group suggests that in the first few days after commencing a VLED there may actually be an increase in steatosis and reduced diastolic filling. ${ }^{36}$

There is a wide range of research incorporating the use of LED/VLED; however, the definition in practice is somewhat blurred, with VLED often much lower than $800 \mathrm{kcal} /$ day. It is recognised that weight loss results from LED around $800 \mathrm{kcal} /$ day are similar to those with lower energy intakes but with fewer adverse effects including limited loss of lean mass. ${ }^{37}$ For this study, we therefore chose a diet based on an intake of $\approx 810 \mathrm{kcal} /$ day (Cambridge Weight Plan).

\section{Exercise programmes}

Physical activity has consistently been associated with a reduced risk of T2D and a reduced risk of premature mortality and CVD mortality in those with diagnosed T2D. ${ }^{38} 39$ Interventional studies have demonstrated a strong causal link between exercise training and glycaemic control in those with T2D. Indeed, exercise training has consistently been found to lower HbAlc by $0.6 \%-0.7 \%$, with greater effects seen with higher volumes of exercise. ${ }^{40}$ Importantly, these substantial benefits are maintained when exercise training does not result in weight loss. ${ }^{40}{ }^{41}$ This is consistent with experimental studies that have elucidated key insulin-dependent and insulin-independent pathways linking physical activity to improved glucose regulation that do not act through adiposity. ${ }^{42} 43$ Therefore, physical activity is an effective therapy for promoting metabolic health and glucose regulation independent of body weight. While the benefits of exercise training on glycaemic control are well established, the effects on diastolic function are less well known, primarily due to insufficient data, differences in measurement and poor study design. However, encouraging data are starting to emerge in those with obesity. For example, an 8-week exercise training programme in obese men improved diastolic function to levels seen in lean controls, despite no weight loss. ${ }^{44}$ Similarly, just 4 weeks of exercise training has been shown to improve diastolic function in those with HF with similar improvements also observed in a matched cohort without $\mathrm{HF}^{45}$
Only two studies that we are aware of have been conducted specifically in people with T2D assessing the effects of exercise training on diastolic function. A small pilot study found improvements in diastolic function following 3 months of aerobic exercise training in those with T2D (although tissue Doppler imaging was not performed, and the randomised groups were not well matched) ${ }^{46}$ while another study found no overall effect. ${ }^{47}$ However, in the latter study, a post hoc analysis revealed that change in moderate-intensity physical activity was significantly associated with change in myocardial strain rate, although it is unclear whether this was systolic, diastolic or both. ${ }^{47}$ This mirrors the wider evidence where light-to-moderate aerobic exercise training has repeatedly been demonstrated to improve diastolic function across a number of groups. ${ }^{446}$ The effectiveness of vigorous-intensity exercise or combined aerobic and resistance training is less well established, with at least one study showing the latter approach is not effective. ${ }^{48}$ Given this evidence base, it is important the efficacy of aerobic exercise training is investigated further, including in younger adults with T2D.

\section{Aims}

The primary aim of the study is to determine if diastolic function can be improved by either a meal replacement low energy diet plan (MRP) or a supervised exercise programme, compared with guideline-directed care. Key secondary aims are to assess the effects of the interventions on glycaemic control and insulin resistance, exercise capacity, blood pressure, changes in body composition and association of favourable cardiac remodelling with improvements in weight loss, exercise capacity and glycometabolic control. The primary hypothesis is that an MRP and/or exercise will improve diastolic function when compared with standard care alone.

\section{METHODS AND ANALYSIS \\ Study design}

This is a single-centre study, which will comprise a baseline and follow-up analysis of a three-arm 12-week prospective, randomised, open-label, blinded end point trial of a low-energy MRP and an exercise programme compared with standard care in young adults with T2D.

\section{Study population}

Potential participants will be identified from both primary and secondary care in Leicester, Leicestershire and Rutland using electronic databases and with support from the National Institute for Health Research East Midlands Local Clinical Research Network. At-risk groups, such as slimming clubs/weight watchers, will also be contacted within the local community. All patients meeting the inclusion and exclusion criteria (table 1) will proceed to the baseline visit and the study. We describe our age range as 'younger' and not specifically as 'young' as in developed countries, diabetes prevalence peaks between 
Table 1 Inclusion and exclusion criteria

\begin{tabular}{|c|c|}
\hline Inclusion criteria & Exclusion criteria \\
\hline $\begin{array}{l}\text { Capacity to provide informed consent before any trial- } \\
\text { related activities. } \\
\text { Established T2D ( } \geq 3 \text { months). } \\
\text { HbA1c } \leq 9 \% \text { if on triple therapy or } \leq 10 \% \text { on diet and } \\
\text { exercise or monotherapy or dual therapy. } \\
\text { Current glucose-lowering therapy either mono, dual or triple } \\
\text { of any combination of metformin, sulphonylurea, DPP-IV } \\
\text { inhibitor, GLP-1 therapy or an SGLT2i } \pm \text { diet and exercise. } \\
\text { Poorly managed diet controlled diabetes (with } \\
\text { HbA1c }>6.5 \% \text {, not currently taking any glucose-lowering } \\
\text { therapy, meeting BMI inclusion range). } \\
\text { BMI }>30 \mathrm{~kg} / \mathrm{m}^{2} \text { or }>27 \mathrm{~kg} / \mathrm{m}^{2} \text { (South Asian). } \\
\text { Diagnosis of } \mathrm{T} 2 \mathrm{D} \text { before the age of } 60 \text { years of age. } \\
\text { Age } \geq 18 \text { and } \leq 65 \text { years. }\end{array}$ & $\begin{array}{l}\text { Diabetes duration }>12 \text { years. } \\
\text { Currently taking more than three glucose-lowering } \\
\text { therapies. } \\
\text { Weight loss of }>5 \mathrm{~kg} \text { in the preceding } 6 \text { months. } \\
\text { Stage } 4 \text { or } 5 \text { chronic kidney disease (eGFR }<30 \mathrm{~mL} \\
\text { min } / 1.73 \mathrm{~m}^{2} \text { ). } \\
\text { Current therapy with insulin, thiazolidinediones, steroids or } \\
\text { atypical antipsychotic medication. } \\
\text { Untreated thyroid disease. } \\
\text { Known macrovascular disease including coronary artery } \\
\text { disease, stroke/TIA or peripheral vascular disease. } \\
\text { Presence of arrhythmia (including atrial fibrillation, atrial } \\
\text { flutter or second or third degree atrioventricular block). } \\
\text { Known heart failure or other clinically relevant heart disease. } \\
\text { Inability to exercise or undertake a MRP. } \\
\text { Absolute contraindication to CMRI. } \\
\text { Cardiovascular symptoms (angina and limiting dyspnoea } \\
\text { during normal physical activity). } \\
\text { Inflammatory condition, for example, connective tissue } \\
\text { disorder and rheumatoid arthritis. }\end{array}$ \\
\hline
\end{tabular}

BMI, body mass index; CMRI, cardiovascular MRI; DPP-IV, dipeptidyl peptidase-IV inhibitor; eGFR, estimated glomerular filtration rate; GLP-1, glucagon-like peptide 1; MRP, meal replacement plan; SGLT2i, sodium glucose cotransporter 2 inhibitor; T2D, type 2 diabetes; TIA, transient ischaemic attack.

the ages of 75 years and 79 years. ${ }^{49}$ In this study, the inclusion of people with $\mathrm{T} 2 \mathrm{D}<65$ years therefore classifies the cohort as younger relative to the general UK population of people with T2D. A Consolidated Standards of Reporting Trials (CONSORT) diagram will be produced, which will include the number of patients: approached, consenting, meeting eligibility criteria, randomised and completing each phase of the study.

\section{Study visits}

An outline of study visits and measures taken is shown in figure 1. Participants are offered $£ 50$ compensation for each of the three assessment visits completed (baseline, 4-week and 12-week visits).

\section{Baseline visit and randomisation}

A study clinician will check eligibility of the potential participant and obtain written informed consent. Baseline data including patients' demographics, medical history and anthropometric measures (height, weight, body mass index, blood pressure and waist and hip measurements) will be collected. Total fat mass and fat free mass will be assessed by a dual-energy X-ray absorptiometry (DEXA) scan (Lunar iDEXA, General Electric Healthcare, Bedford, UK). Habitual physical activity and sedentary time will be assessed using objective (ActiGraph GT3X+ accelerometer, USA) and subjective (physical activity questionnaires) measures. A fasting blood sample will also be taken to check a range of measures including a biochemical profile for diabetes control, liver and kidney function and lipid profile, together with other measures of endocrine function (eg, fasting insulin,
C-peptide and thyroid function tests) and inflammation. Insulin resistance will be estimated using the homeostatic model assessment-insulin resistance method mo $^{50}$ and the triglyceride/high-density lipoprotein (HDL) ratio. ${ }^{51}$ Additional blood samples will be collected for the storage of plasma and serum for future metabolomic and proteomic investigation.

Participants will undergo comprehensive CMRI scanning and transthoracic echocardiography performed at baseline as outlined below. They will then undergo a maximum symptom-limited incremental exercise test on a stationary electromagnetically braked cycle ergometer (eBike, General Electric Healthcare, Bedford, UK) with expired gas analysis (Ganshorn PowerCube, General Electric Healthcare, Bedford, UK) to determine peak oxygen consumption (oxygen consumption $\left(\mathrm{VO}_{2}\right)$ ), the gold standard assessment of cardiovascular fitness. ${ }^{52}$

\section{Randomisation}

Participants will undergo randomisation at the baseline visit, using an independent online computerised randomisation system (Sealed Envelope). Randomisation will be stratified by sex, given different LV remodelling processes in men and women, ${ }^{53}$ and by baseline glucose-lowering therapy (any glucagon-like peptide-1, dipeptidyl peptidase-IV or sodium glucose cotransporter 2 inhibitor vs none of these).

\section{Study interventions}

Recruited participants will be randomised to one of three groups: (1) standard of care as per National Institute for 


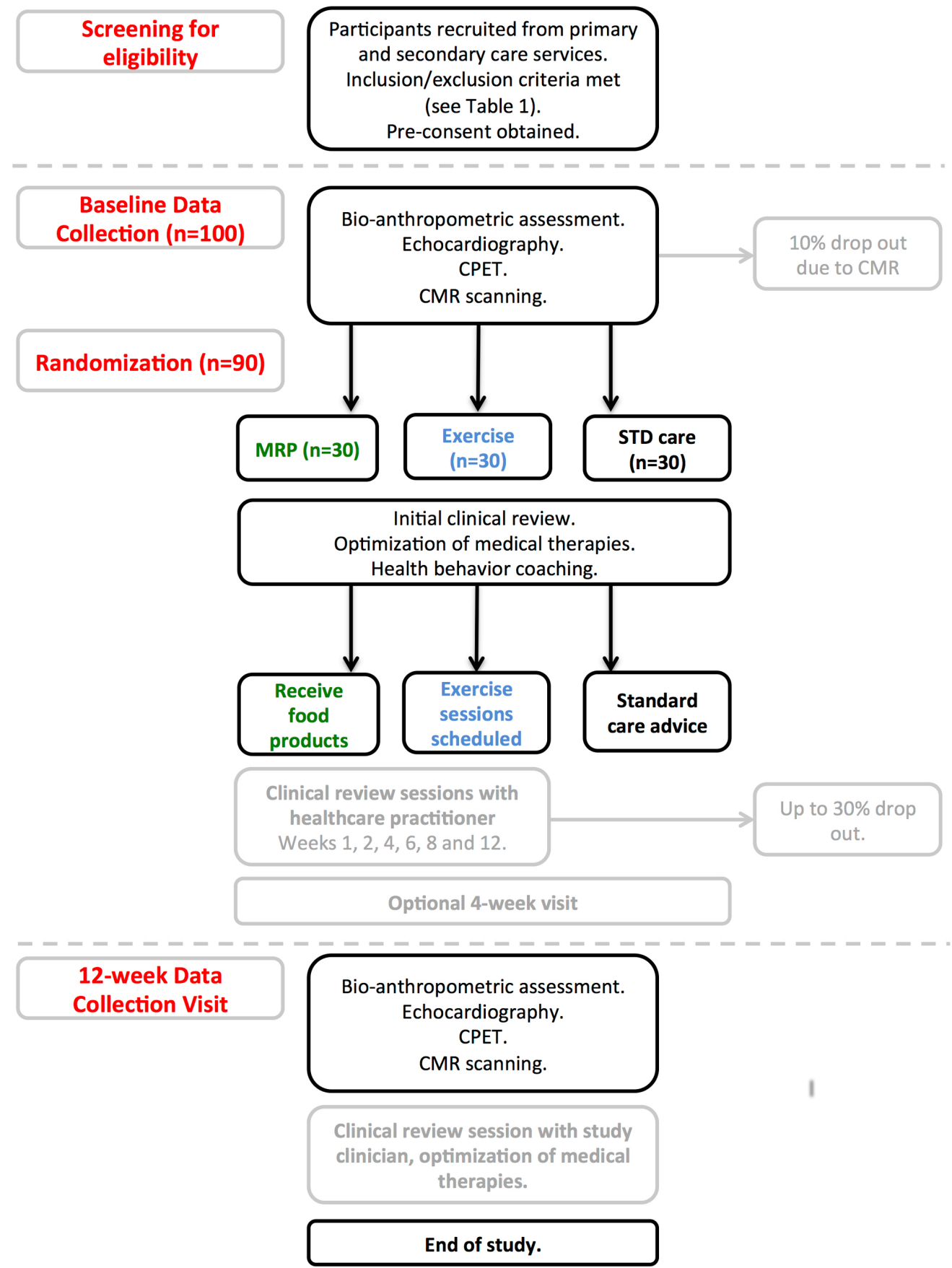

Figure 1 Study overview. CMR, cardiac MRI; CPET, cardiopulmonary exercise testing; MRP, meal replacement plan MRP.

Health and Care Excellence guidance ${ }^{54}$ including lifestyle advice, (2) MRP or (3) supervised exercise training.

1. The standard care group will be provided with standard lifestyle advice according to National Institute for Health and Care Excellence guidance,$^{55}$ delivered in a single health coaching interview at week 0 . This lifestyle advice will be reinforced at the week 12 assessment.

2. The MRP group will receive a low-energy MRP diet containing an average of $\approx 810 \mathrm{kcal} /$ day $(30 \%$ protein, $50 \%$ carbohydrate, $20 \%$ fat) (Cambridge Weight Plan). This diet plan complies with all current guidance and government legislation (European Food Safety
Authority) for macronutrient and micronutrient content and quality. ${ }^{56}$ Participants randomised to the MRP group will be asked to discontinue all glucose-lowering therapies following randomisation to avoid hypoglycaemia. Antihypertensive drugs will be stopped on the day of low-energy diet commencement, as a safety measure because blood pressure is likely to fall substantially (mean drop in systolic blood pressure $\sim 20 \mathrm{~mm} \mathrm{Hg}$ ) on the diet. ${ }^{35}$ Any alterations to medication will ultimately be made at the discretion of the study clinician(s). The clinical team will include specialists in CVD and diabetes medicine to oversee alterations in antihypertensive and glucose-lowering therapy. 
Blood pressure and glucose will be monitored throughout the study at the clinical review sessions (see below).

The MRP will be undertaken alongside health behaviour coaching and relapse prevention through weekly contact, where possible, with a qualified dietician or equivalent. Participants will be asked to maintain their usual daily activities and not initiate any additional physical activity for the duration of the study. Those participants in the MRP arm who do not achieve a loss of $>2 \%$ body weight at week 1 and $4 \%$ at week 3 will be considered non-compliant and excluded from the study. Investigator discretion will be used when considering non-compliance in the event of borderline values. The diet will be stopped, and a maintenance diet reintroduced once $50 \%$ excess body weight has been lost, or by 12 weeks, whichever comes first. The maintenance diet will be individualised and agreed with the participant, based on their estimated total energy expenditure at that point. The self-reported intake at week 12 will be recorded and comparisons made between arms for macronutrient and energy intake.

3. The exercise group will attend supervised sessions at the Leicester Biomedical Research Centre. The exercise programme will be undertaken thrice weekly, progressing to $50 \mathrm{~min}$ per session of moderate intensity aerobic exercise, in line with prevailing guidelines (150 min per week). Each exercise session will consist of a warm-up, stimulus and cool-down phase. Static stretching will be undertaken at the end of each session. The stimulus phase will include walking and/or lower extremity cycling. The duration of exercise in each training mode will also be increased gradually to achieve the 50 min target. An initial assessment of cardiorespiratory fitness will be performed, and exercise intensity titrated using the value from the baseline peak $\mathrm{VO}_{2}$ assessment. Objective (heart rate monitoring) and subjective (Borg Rate of Perceived Exertion Scale, graded from 6 to 20) measures will be used to measure responses to the exercise sessions with exercise intensity adjusted progressively to take account of increasing fitness levels throughout the intervention period as follows:

Weeks 1 and 2: $15 \mathrm{~min}$ at $50 \%$ peak $\mathrm{VO}_{2}$.

Week 3: 25 min at $\sim 60 \%$ peak $\mathrm{VO}_{2}$.

Week 4: 30 min at $\sim 60 \%$ peak $\mathrm{VO}_{2}$.

Week 5: 35 min at $\sim 60 \%$ peak $\mathrm{VO}_{2}$.

Week 6: 40 min at $\sim 60 \%$ peak $\mathrm{VO}_{2}$.

Week 7: $45 \mathrm{~min}$ at $\sim 60 \%$ peak $\mathrm{VO}_{2}$.

Weeks 8-12: 50 min at $\sim 60 \%$ peak $\mathrm{VO}_{2}$.

Although weight loss may occur in the exercise group, this is not the main aim of the intervention, which is to assess whether diastolic function improves independent of weight loss. Compliance will be assessed by attendance at the supervised sessions. Patients in the exercise arm who attend less than two-thirds of the supervised sessions in the first 4 weeks and then throughout the remainder of the study period will be excluded. Investigator discretion may be used in cases where compliance is borderline.

Participants will be asked to maintain their usual dietary intake, and a self-reported food diary will be applied before and after the intervention to estimate intake.
Participants in the exercise arm will have their medication reviewed by the study clinician at baseline. Those with an HbAlc $\leq 8 \%$ who are currently taking a sulphonylurea will initially have the dose reduced by $50 \% 72$ hours prior to the first exercise session. Their regimes will be continued to be down-titrated starting with the sulphonylurea and then moving, where applicable, onto their current sodium glucose cotransporter-2 inhibitor or dipeptidyl peptidase-IV inhibitor or glucagon-like peptide-1 therapies according to glycaemic control. The latter therapies will be titrated down last given their relative low risk of hypoglycaemic events. For those participants with an HbAlc $\leq 8 \%$ who are not taking a sulphonylurea, the study clinician will down-titrate glucose-lowering therapy on an individual basis, taking into account the class and combination of therapies prescribed. All other medication will be maintained unless the study clinician deems alterations are necessary for a patient's best interest. Alterations in medication will be supervised by clinicians specialised in diabetes medicine.

\section{Subsequent clinical reviews}

All participants will have an initial clinical review postrandomisation at the time of their health coaching. In order to ensure their continued safety, each participant in the MRP or exercise arm will be invited for frequent clinical reviews. They will attend six reviews after randomisation in total (weeks 1, 2, 4, 6, 8 and 12), as well as receiving a telephone consultation at weeks 3 and 10 . At these clinical reviews their blood pressure, fasting glucose (weeks 1 , 2 and 4 for MRP arm) and weight will be measured. This is to inform: (A) any additional alterations to medication that need to be made and (B) compliance to the MRP and exercise programme as appropriate.

\section{Optional 4-week visit}

The 4-week assessment is optional and will only be conducted on a subset of participants who provide additional consent. Measures taken during the baseline visit will be repeated to ascertain whether there are early alterations to outcome measures in participants randomised to the study interventions.

\section{Twelve-week final data collection visit}

All measures and tests undertaken at the baseline visit will be repeated at the 12-week final data collection visit. Participants will undergo one further clinical review (see above) following the 12-week data collection visit, which will be the final visit and the end of the study. Medications will be reviewed, and any medication reintroduced will be tailored to the individual based on previous and current HbAlc and blood pressures. This review will be undertaken by an experienced study clinician and the outcome communicated to the patient's usual physician and general practitioner.

\section{Blood glucose monitoring}

Participants in both intervention arms (MRP and exercise) will be provided with blood glucose monitoring 
equipment to capture seven-point glucose profiles across key time-points during the study (prior to initial consultation week 0 , prior to the 4 -week visit and prior to 12 -week visit), which will be recorded in a blood glucose monitoring diary and/or via direct download of the blood glucose metre data at the end of the study where possible. It will also allow them to self-monitor their glucose levels through the study and capture any hypoglycaemic events to guide medication adjustments accordingly if required. Additionally, hyperglycaemia will be assessed, and medication adjusted if necessary after review of compliance to intervention. These will be self-reported by the participant in the blood glucose monitoring diary.

\section{Magnetic resonance imaging}

Reproducibility of PEDSR appears to be better at $1.5 \mathrm{~T}$ than at $3 \mathrm{~T}$, and therefore, $1.5 \mathrm{~T}$ is our preferred field strength for CMR assessment of diastolic function. ${ }^{57}$ Myocardial triglyceride on the other hand is measured using magnetic resonance spectroscopy, a technique generally considered to be best performed at 3T, due to the higher signal to noise ratio compared with $1.5 \mathrm{~T}^{58}$ We will therefore perform magnetic resonance spectroscopy on a 3T scanner (Skyra, Siemens, Erlangen), with all other MRI data being collected on a $1.5 \mathrm{~T}$ scanner (Aera, Siemens, Erlangen).

The MRI protocol is outlined in figure 2 and is similar to that described previously. ${ }^{59}$ An exception to this is that myocardial tissue tagging and T1 mapping have been omitted. Myocardial tissue tagging requires the acquisition of additional images with long breath hold duration and is time consuming to analyse. Alternatives include phase velocity mapping, displacement encoding with simulated echoes and strain-encoded images, all of which require the acquisition of additional sequences and/or involve lengthy postprocessing times. ${ }^{60}$ Instead, we will use tissue tracking of balanced steady-state free precession LV cine images, which has emerged as a reliable alternative technique for the assessment of strain and diastolic strain rate $e^{576162}$ with good test-test reproducibility for PEDSR in our lab. ${ }^{56}$

Briefly, after localisers, Dixon gradient echo pulse sequences covering the thorax and abdomen will be acquired for thoracic and abdominal subcutaneous and visceral fat. Liver triglyceride content will also be assessed by HepaFat-Scan, which has been validated against stereological analysis of liver biopsy sections for measurement of liver fat fraction. ${ }^{63}$ Steady-state free precession cine images will be acquired in four-chamber, threechamber and two-chamber views. Perfusion images will be acquired after pharmacological vasodilation with adenosine $(140 \mu \mathrm{g} / \mathrm{kg} / \mathrm{min}$, for 2-3 min) until a haemodynamic response is achieved (a drop in systolic blood pressure of $10 \mathrm{~mm} \mathrm{Hg}$ and/or rise in heart rate of 10 bpm). During peak stress, a gadolinium-based contrast agent (Gadoterate meglumine, Dotarem, Guerbet LLC, France) will be injected $(0.04 \mathrm{mmol} / \mathrm{kg})$, followed by a $20 \mathrm{~mL}$ bolus of normal saline, at a rate of $5 \mathrm{~mL} / \mathrm{s}$, and perfusion images will be acquired using a saturation recovery gradient echo pulse sequence for three slices (basal, mid and apical). Rest imaging will be performed approximately $10 \mathrm{~min}$ after stress imaging with a further $0.04 \mathrm{mmol} / \mathrm{kg}$ of contrast. In the intervening time, a stack of short-axis slices will be obtained using cine images covering the entire LV. A further $0.07 \mathrm{mmol} / \mathrm{kg}$ top-up of contrast will be administered to bring the total contrast dose to $0.15 \mathrm{mmol} / \mathrm{kg}$. Aortic cine images will be acquired in a plane perpendicular to the thoracic aorta at the level of the pulmonary artery bifurcation. Through-plane phase contrast sequences perpendicular to the ascending and descending aorta at the level of the pulmonary bifurcation will be captured for pulse wave flow analysis. To determine the aortic arch segment length between which aortic pulse wave velocity is calculated, sagittal oblique aortic cine images will be acquired. At least 10 min after top-up contrast administration, late gadolinium enhancement images will be obtained with an inversion-recovery prepared, segmented gradient echo sequence.

\section{CMRI analysis}

Scans will be anonymised and sent to a standalone workstation for blinded analysis as previously described. ${ }^{19}$ Briefly systolic strain and longitudinal PEDSR will be quantified from cine images using tissue tracking. LV volumes, mass and function will be calculated using commercially available software (cmr42, Circle Cardiovascular Imaging, Calgary, Canada). Myocardial strain will be measured using both Image Arena Feature Tracking (TomTec Imaging Systems, Unterschleissheim, Germany) and cmr42 Tissue Tracking (Circle Cardiovascular Imaging). Interobserver and intraobserver variability will be calculated on at least 10 random datasets by two experiences observers and results reported. Test-retest reproducibility will be assessed for the primary endpoint in 10 subjects and the analysis technique that has the best reproducibility will be used for the final analysis, and if they have similar reproducibility, we will use tissue tracking since this can be generated using the contours drawn for LV mass analysis. The epicardium and endocardium will be contoured on the perfusion images, along with a region of interest in the LV blood pool to generate signal intensity curves. Myocardial perfusion reserve will be calculated using Fermi-constrained deconvolution as previously described. ${ }^{19}$ Late gadolinium enhancement will be assessed qualitatively as absent or present and quantified by a thresholding technique $>5$ SDS above remote normal myocardium. ${ }^{64}$

\section{Transthoracic echocardiography}

Comprehensive transthoracic echocardiography will be performed according to British Society of Echocardiography guidelines. ${ }^{65}$ Each participant will have assessment for: tissue Doppler indices of diastolic filling and speckle tracking for systolic and diastolic strain/strain rate, exclusion of valvular abnormalities, assessment of $\mathrm{LV}$ size and function. Given the difficulties in grading diastolic dysfunction in mild and moderate disease, ${ }^{66}$ the primary focus on echocardiography will be on $\mathrm{E} / \mathrm{e}^{\prime}$, a non-invasive surrogate 

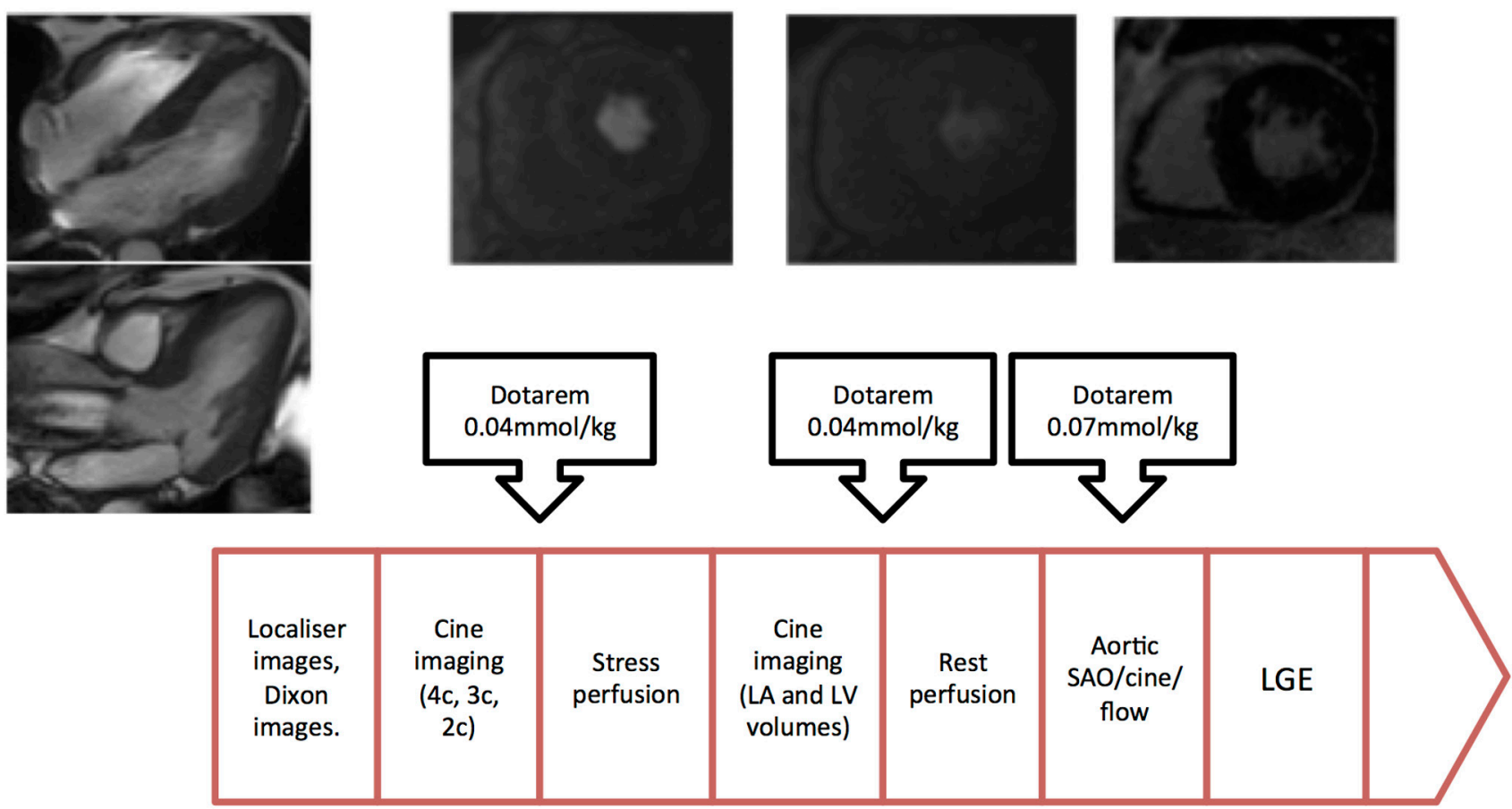

\begin{tabular}{|l|l|l|l|l|l|}
\hline 5 mins & 5 mins & 5 mins & $10 \mathrm{mins}$ & $2 \mathrm{mins}$ & $10 \mathrm{mins}$ \\
\hline
\end{tabular}

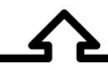

Adenosine $140 \mu \mathrm{g} / \mathrm{kg} / \mathrm{min}$
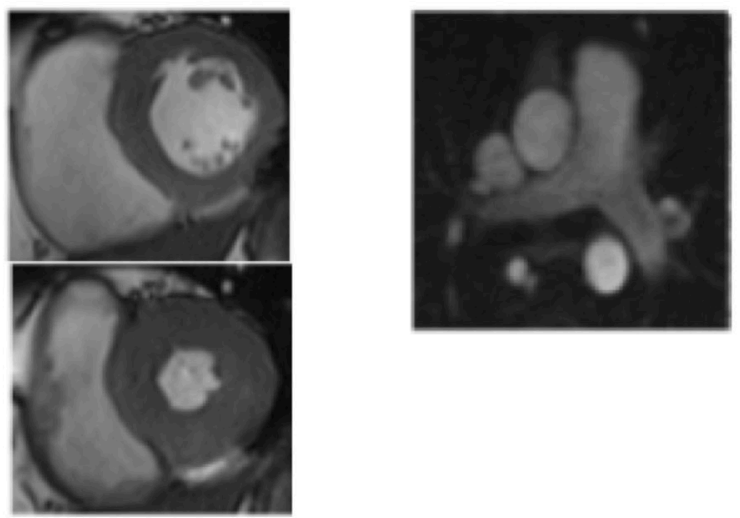

Figure 21.5 Tesla CMR protocol for covering thoracic and abdominal subcutaneous and visceral fat (Dixon) function (cine), arterial stiffness and late gadolinium enhancement for focal fibrosis. CMR, cardiac MRI; LA, left atrium; LGE, late gadolinium enhancement; LV, left ventricle; SAO, sagittal oblique.

of LV filling pressure. ${ }^{67}$ Mitral annular velocities $\left(\mathrm{e}^{\prime}\right)$ will also be reported to account for the possibility that enlarged left atrial volumes may result in a decreased $\mathrm{E}$ wave velocity and apparent reduction of $\mathrm{E} / \mathrm{e}^{\prime}$ in people with diabetes. ${ }^{68}$

\section{Study outcomes}

\section{Primary outcome}

The primary outcome measure is change in circumferential PEDSR, measured by CMR from baseline to 12 weeks. Other measures of cardiac structure and function assessed in the study are shown in box 1 .

\section{Secondary outcomes}

Anthropometric variables

Body weight, height, hip and waist circumference will be measured to the nearest $0.1 \mathrm{~kg}, 1 \mathrm{~cm}, 1 \mathrm{~cm}$ and $1 \mathrm{~cm}$, respectively. Waist circumference will be measured using a soft tape with the participant standing, feet together, at the level of the umbilicus. Hip circumference will be measured at the level of the greater trochanters. Brachial blood pressure will be obtained from the seated participant's dominant arm. Three measurements will be taken 


\section{Box 1 Key imaging outcome measures}

Primary outcome measures

- Change in circumferential PEDSR measured from baseline to 12 weeks.

\section{Secondary outcome measures \\ - Longitudinal PEDSR. \\ - Standard assessments of structure and function (eg, LV mass, end-diastolic/systolic volumes, mass/volume and ejection fraction). \\ - Left atrial volumes. \\ - Myocardial systolic strain/strain rates (circumferential and longitudinal). \\ - Arterial stiffness (mean ascending and descending aortic distensi- bility and pulse-wave velocity). \\ - Global myocardial perfusion reserve as a marker of microvascular function. \\ - Myocardial and liver triglyceride content. \\ - Echocardiographic mitral inflow velocity $(E)$, myocardial relaxation $\left(E^{\prime}\right)$ and $E / E^{\prime}$, a non-invasive marker of $L V$ filling pressure. Diastolic dysfunction will be graded.}

LV, left ventricular; PEDSR, peak early diastolic strain rate.

and an average of the last two measurements will be used. Ethnicity, smoking status, medical history and medications history will be obtained by self-report.

\section{Biochemical variables}

Standard biochemical outcomes will be assessed by venous blood sampling. These will include fasting glucose and HbA1c, lipid profile (fasting triglycerides, HDL, low-density lipoprotein and total cholesterol), urea and electrolytes (sodium, potassium, urea and creatinine) and liver function tests (albumin, total bilirubin, alkaline phosphatase and alanine transaminase). Postmeal increase of glucose and absolute values of postmeal glucose will be assessed from the seven-point glucose profile.

\section{Cardiovascular fitness}

Participants will undergo a maximum incremental exercise test on a stationary bicycle (electromagnetically braked cycle ergometer) with expired gas analysis to determine peak $\mathrm{VO}_{2}$. The exercise test will begin with a 3 min warm-up phase and end with a 3 min cool-down phase. One-minute workload increments will be based on patient age, gender, height and weight. ${ }^{52}$ The test will be physician supervised, and blood pressure will be recording at 2 min intervals. Indications for medical termination will be as previously described. ${ }^{69}$ A quality control cardiopulmonary exercise test is undertaken every 6 weeks using a biological control in our unit. The coefficient of variation for $\mathrm{VO}_{2}(\mathrm{~L} / \mathrm{min})$ during steady state at workloads of 75 , 100,125 and 150 watts is consistently $<8 \%$.

\section{Recent Physical Activity Questionnaire}

Self-reported physical activity will be measured using the Recent Physical Activity Questionnaire as previously described. ${ }^{70}$ This assesses physical activity across four domains (domestic, recreational, occupational and commuting) over the previous month. The Recent Physical Activity Questionnaire has been shown to be a reliable and valid tool for measuring physical activity. ${ }^{71}$

\section{Accelerometer}

Participants will be asked to wear the accelerometer on a waistband (in the right anterior axillary line) during waking hours for seven consecutive days. A log sheet is provided for participants to record each day that they wear the accelerometer and document when the accelerometer was removed at night, time they went to sleep, time they woke up and time the accelerometer was reattached. Participants will return the accelerometer and log sheet via prepaid envelope at the end of 7 days of wear. A minimum of 3 days valid wear will be required to count as a valid recording. Raw acceleration data are captured and stored at $100 \mathrm{~Hz}$. Data processing will be undertaken on commercially available analysis software.

\section{Dual-energy X-ray absorptiometry}

Each participant will undergo a DEXA scan using a totalbody scanner. DEXA uses a technology by which the radiation at two energies is used to determine components of the attenuating tissue, either bone and soft tissue or lean soft tissue and fat. DEXA has been validated as a measure of body fat in obese and normal weight individuals ${ }^{72} 73$ and as such is considered to be one of the gold standard measurements for determining body composition.

Estimates of total and regional fat mass and fat free mass will be automatically derived using the iDEXA encore software. Areas of particular interest include the appendages and trunk (encompassing android and gynoid regions). Daily quality assurance and quality controls will be carried out during the study period according to standard procedures supplied by the manufacturer. The volunteers will be scanned using standard imaging and positioning protocols.

\section{Statistical analyses}

\section{Sample sizes}

The power calculation is based on the previously mentioned pilot study from our group. ${ }^{19}$ The patients with T2D had a circumferential PEDSR of $1.5 \frac{1}{\mathrm{~s}}$ with SD of $0.2 \frac{1}{\mathrm{~s}}$. Based on this, 30 patients per group will have $80 \%$ power to detect a between group difference in PEDSR of $0.2 \frac{1}{\mathrm{~s}}$ assuming alpha $=0.025$ (to allow for two primary comparisons, ie, MRP vs standard care and exercise vs standard care) and a maximum $30 \%$ dropout. ${ }^{29} 30$ Such an improvement would result in a strain rate $\left(1.7 \frac{1}{\mathrm{~s}}\right)$ similar to that seen in the obese controls $\left(1.8 \frac{1}{\mathrm{~s}}\right)$ but still lower than the lean controls $\left(2.0 \frac{1}{\mathrm{~s}}\right)$.

\section{Statistical methods and analyses}

The aim of these analyses is to compare the change in primary outcome (PEDSR) at 12 weeks between the MRP and standard care arms, and between the exercise and standard care arms. The primary analysis will be a complete case analysis as this is a proof of principle study where we are primarily interested in the size of 
the treatment effect, rather than the practicability of the intervention. A CONSORT diagram will be produced. At baseline and each follow-up, descriptive variables and changes in outcomes will be summarised by arm using mean (SD) for normally distributed continuous variables, median (IQR) for non-normally distributed continuous variables and count (percentage) for categorical variables. Each intervention will be compared with the control group (ie, MRP vs standard care and exercise vs standard care) using linear regression adjusted for stratification factors and baseline values. The exercise and MRP arms will be compared as a secondary analysis. If necessary, the outcome variable will be appropriately transformed so that the assumptions of the linear regression model are held. Intention-to-treat analyses will be performed as sensitivity analyses using multiple imputation or another appropriate approach to impute missing data.

\section{Patient and public involvement (PPI)}

The University of Leicester and the Leicester NIHR Biomedical Research Centre have very active PPI groups in both diabetes and cardiovascular sciences. The study outline was presented to our PPI groups for feedback prior to designing the study protocol. In addition, PPI focus groups were held about the study design and conduct before the protocol was finalised. In particular, the PPI groups assessed the burden of the exercise and acceptability of the MRP arms on study participants to ensure appropriate support was available to help compliance with these interventions. Furthermore, the trial steering committee (see below) has at least two lay members for study oversight. All study participants and members of the public will be invited to return for a lecture disseminating the study's key findings on completion.

\section{Trial oversight and governance}

The study sponsor is the University of Leicester and is managed by the Leicester Clinical Trials Unit (CTU) and is overseen by the trial steering committee composed of an independent chair and a group of experts. The independent chair of the trial steering committee is Professor Roy Taylor, professor of medicine and metabolism and director of the Magnetic Resonance Centre, Newcastle University. This study is registered on http://www.clinicaltrials.gov and monitored by the University of Leicester. Data completeness and quality is reviewed by the Leicester CTU. The quality of 10 randomly selected baseline MRI datasets was assessed by an independent laboratory (Professor Sven Plein, Leeds, UK) and all images were graded as excellent or good.

Given the open design of the study, it was determined that a data monitoring and ethics committee was not required. All serious adverse events are reported to the sponsor according to current guidelines and the trial steering committee.
Current status and timescale

Recruitment started in January 2016 and follow-up of the last participant was completed by end of August 2018 .

\section{DISCUSSION}

Younger adults with T2D are at very high lifetime risk of developing HF. Concentric LV remodelling with associated diastolic dysfunction appears to be the earliest manifestation of diabetic cardiomyopathy. The occurrence of diastolic dysfunction reflects abnormal LV relaxation and increased LV stiffness and has been shown to predict an increased risk of developing overt HF within 5 years. ${ }^{74}$ Reported prevalence rates of diastolic dysfunction in asymptomatic patients with T2D are as high as $78 \%{ }^{75}$ Therapies that may reverse diastolic dysfunction or prevent progression to overt chronic HF are therefore urgently needed.

In the DiRECT study delivered in primary care, an MRP programme led to an average weight loss of $10 \mathrm{~kg}$ and remission of diabetes in $46 \%$ of overweight or obese patients with T2D. ${ }^{27}$ The cardiovascular benefits of weight loss, however, are less well characterised in T2D, although a reduction in myocardial steatosis and improved diastolic filling has been shown with a VLED in T2D. ${ }^{35} \mathrm{~A}$ more substantial body of evidence exists for the beneficial cardiovascular effects of weight loss in obese non-diabetic subjects including: reductions in mass/volume, improved diastolic dysfunction and arterial stiffness. ${ }^{33} 76$

Similarly, exercise training has favourable effects on glycaemic control and cardiovascular outcomes in T2D. ${ }^{38-41}$ These benefits of exercise training appear independent of weight loss, suggesting that the underlying mechanisms are different. ${ }^{40} 41$ However, data supporting positive effects of exercise training on diastolic dysfunction in T2D are lacking, although reversal of diastolic dysfunction with exercise has been demonstrated in subjects with obesity. ${ }^{44}$ This study will be among the first to compare and establish the effects of weight loss and exercise on cardiac structure and function in younger adults with T2D. If either of these interventions were to successfully reduce diastolic dysfunction, it is likely that the outcome will contribute to national and international guidelines in this area.

\section{Other interventions that may improve diastolic dysfunction}

Newer glucose-lowering therapies may improve cardiovascular (CV) outcomes in patients with T2D. Sodium glucose cotransport-2 inhibitors reduce the risk of HF and glucagon-like peptide-1 receptor agonists lower thrombotic events, ${ }^{77}$ but for optimum benefit, they will require to be used in conjunction with lifestyle interventions, which may actually modify the underlying pathophysiological process of T2D development. Similarly, intensive blood pressure lowering may or may not improve CV outcomes in patients with T2D, but there are inconsistencies in the recommended blood pressure targets. ${ }^{55} 7879$ Current National Institute for Health and Care Excellence 
(NG28) recommendations are that blood pressure be maintained below $140 / 80 \mathrm{~mm} \mathrm{Hg}$ in uncomplicated T2D or below $130 / 80 \mathrm{~mm} \mathrm{Hg}$ if there is a history of kidney, eye or cerebrovascular disease. ${ }^{55}$ Importantly, both MRP $\operatorname{diets}^{31} 35$ and exercise ${ }^{8081}$ have favourable effects on blood pressure, supporting these strategies as interventions for CV risk reduction in T2D.

\section{Limitations}

This is a proof-of-efficacy study and therefore effects on clinical outcomes are not planned with a short duration of follow-up. Only patients who are able to exercise and willing to undertake the MRP are eligible, and we have limited our inclusion to younger adults ( $<65$ years) with a maximum duration of diabetes of 12 years, so the results will not necessarily be generalisable to all people with T2D. The study interventions, given their nature, are not blinded, but ascertainment bias is limited by the blinded assessment of the primary outcome and other imaging parameters. Only the core lab is performing the quantitative analysis of blinded images, and we will not request that another laboratory performs independent segmentation analysis for evaluation of interlaboratory variation. However, a subset of 10 randomly selected CMR datasets was reviewed by an internationally regarded independent expert (Professor Sven Plein, Leeds, UK) to assure data quality and integrity. A smaller-than-expected treatment effect in the intervention arms of the study could render the study underpowered, although the current dropout rate is closer to $20 \%$ than the allowed for $30 \%$. The participants in the intervention arms receive increased contact with study investigators that will only partially be offset by health coaching that is administered at baseline to the control group. Follow-up of the last participant recruited to the study was completed by the end of August 2018, which limits changes to data collection.

\section{Declarations}

\section{Ethics and dissemination}

The study is conducted in accordance with the principles of the 1996 Helsinki Declarations, International Conference on Harmonisation-Good Cinical Practice (ICHGCP) guidelines. It is anticipated that data collection will be completed by end of August 2018, and the study results will be submitted for publication within 6 months of completion.

\footnotetext{
Author affiliations

${ }^{1}$ Department of Cardiovascular Sciences, University of Leicester and the Leicester NIHR Biomedical Research Centre, Glenfield Hospital, Leicester, UK ${ }^{2}$ Diabetes Research Centre, University of Leicester and the Leicester NIHR Biomedical Research Centre, Leicester General Hospital, Leicester, UK ${ }^{3}$ National College of Sport and Exercise Medicine, University of Loughborough, Loughborough, UK

${ }^{4}$ University of Leeds, Multidisciplinary Cardiovascular Research Centre and Biomedical Imaging Science Department, Leeds Institute of Cardiovascular and Metabolic Medicine, Leeds, UK
}

Acknowledgements This work would not have been possible without the infrastructure and support of the National Institute for Health Research (NIHR) Leicester Biomedical Research Centre and the Leicester Clinical Trials Unit.

Contributors GPM, MJD, TY and KK conceived the idea for the study. GPMC, EMB, JH and TY designed the study protocol. GSG, EB, EMB and LA drafted the manuscript. EL, GPMC, JVW, KSP and DJS designed the MRI protocol. JM and A-MM designed the echocardiography and cardiopulmonary exercise testing protocols. DB provided statistics support. EB designed the meal replacement diet protocol. JH and TY designed the exercise protocol. All authors critically reviewed and approved the final draft of the manuscript.

Funding This study is funded by the National Institute for Health Research (NIHR) through a career development fellowship (G McCann, CDF 2014-07-045) and directly supported by the NIHR Leicester Biomedical Research Centre.

Competing interests None declared.

Patient consent for publication Not required.

Ethics approval Ethical approval as a clinical trial of an investigational medical product was granted by National Research Ethics Service committee West Midlands (15/WM/0222).

Provenance and peer review Not commissioned; externally peer reviewed.

Open access This is an open access article distributed in accordance with the Creative Commons Attribution Non Commercial (CC BY-NC 4.0) license, which permits others to distribute, remix, adapt, build upon this work non-commercially, and license their derivative works on different terms, provided the original work is properly cited, appropriate credit is given, any changes made indicated, and the use is non-commercial. See: http://creativecommons.org/licenses/by-nc/4.0/.

\section{REFERENCES}

1. Organization, W.H. Diabetes fact sheet. 2017 http://www.who.int/ mediacentre/factsheets/fs312/en/ (cited 5 Mar 2018).

2. Grinstein G, Muzumdar R, Aponte L, et al. Presentation and 5-year follow-up of type 2 diabetes mellitus in African-American and Caribbean-Hispanic adolescents. Horm Res 2003;60:121-6.

3. Wilmot EG, Edwardson CL, Biddle SJ, et al. Prevalence of diabetes and impaired glucose metabolism in younger 'at risk' UK adults: insights from the STAND programme of research. Diabet Med 2013;30:671-5.

4. Haines L, Wan KC, Lynn R, et al. Rising incidence of type 2 diabetes in children in the U.K. Diabetes Care 2007;30:1097-101.

5. Constantino MI, Molyneaux L, Limacher-Gisler F, et al. Long-term complications and mortality in young-onset diabetes: type 2 diabetes is more hazardous and lethal than type 1 diabetes. Diabetes Care 2013;36:3863-9.

6. Schmidt M, Johannesdottir SA, Lemeshow S, et al. Obesity in young men, and individual and combined risks of type 2 diabetes, cardiovascular morbidity and death before 55 years of age: a Danish 33-year follow-up study. BMJ Open 2013;3:e002698.

7. McMurray JJ, Gerstein HC, Holman RR, et al. Heart failure: a cardiovascular outcome in diabetes that can no longer be ignored. Lancet Diabetes Endocrinol 2014;2:843-51.

8. Kannel WB, Hjortland M, Castelli WP. Role of diabetes in congestive heart failure: the Framingham study. Am J Cardiol 1974;34:29-34.

9. Aronow WS, Ahn C. Incidence of heart failure in 2,737 older persons with and without diabetes mellitus. Chest 1999;115:867-8.

10. Bertoni AG, Tsai A, Kasper EK, et al. Diabetes and idiopathic cardiomyopathy: a nationwide case-control study. Diabetes Care 2003;26:2791-5.

11. Petersen S, Rayner M, Westholme J. Coronary heart disease statistics: heart failure supplemen: British Heart Foundation, 2002.

12. Owan TE, Hodge DO, Herges RM, et al. Trends in prevalence and outcome of heart failure with preserved ejection fraction. $N$ Engl $J$ Med 2006;355:251-9.

13. Maisch B, Alter P, Pankuweit S. Diabetic cardiomyopathy-fact or fiction?. Herz 2011;36:102-15.

14. Boyer JK, Thanigaraj S, Schechtman KB, et al. Prevalence of ventricular diastolic dysfunction in asymptomatic, normotensive patients with diabetes mellitus. Am J Cardiol 2004;93:870-5.

15. Kiencke S, Handschin R, von Dahlen R, et al. Pre-clinical diabetic cardiomyopathy: prevalence, screening, and outcome. Eur J Heart Fail 2010;12:951-7.

16. von Bibra $\mathrm{H}$, St John Sutton M. Diastolic dysfunction in diabetes and the metabolic syndrome: promising potential for diagnosis and prognosis. Diabetologia 2010;53:1033-45. 
17. Ceyhan K, Kadi H, Koç F, et al. Longitudinal left ventricular function in normotensive prediabetics: a tissue Doppler and strain/strain rate echocardiography study. J Am Soc Echocardiogr 2012;25:349-56.

18. Yazici M, Ozdemir K, Gonen MS, et al. Is there any relationship between metabolic parameters and left ventricular functions in type 2 diabetic patients without evident heart disease?. Echocardiography 2008;25:675-82

19. Khan JN, Wilmot EG, Leggate $M$, et al. Subclinical diastolic dysfunction in young adults with Type 2 diabetes mellitus: a multiparametric contrast-enhanced cardiovascular magnetic resonance pilot study assessing potential mechanisms. Eur Heart $J$ Cardiovasc Imaging 2014;15:1263-9.

20. Brooks BA, Franjic B, Ban CR, et al. Diastolic dysfunction and abnormalities of the microcirculation in type 2 diabetes. Diabetes Obes Metab 2008;10:739-46.

21. Rijzewijk LJ, van der Meer RW, Smit JW, et al. Myocardial steatosis is an independent predictor of diastolic dysfunction in type 2 diabetes mellitus. J Am Coll Cardiol 2008;52:1793-9.

22. Ng AC, Delgado V, Bertini M, et al. Myocardial steatosis and biventricular strain and strain rate imaging in patients with type 2 diabetes mellitus. Circulation 2010;122:2538-44.

23. Korosoglou G, Humpert PM, Ahrens J, et al. Left ventricular diastolic function in type 2 diabetes mellitus is associated with myocardial triglyceride content but not with impaired myocardial perfusion reserve. J Magn Reson Imaging 2012;35:804-11.

24. Shah AS, Khoury PR, Dolan LM, et al. The effects of obesity and type 2 diabetes mellitus on cardiac structure and function in adolescents and young adults. Diabetologia 2011;54:722-30.

25. Pories WJ, Caro JF, Flickinger EG, et al. The control of diabetes mellitus (NIDDM) in the morbidly obese with the Greenville Gastric Bypass. Ann Surg 1987;206:316-23.

26. Meijer RI, van Wagensveld BA, Siegert CE, et al. Bariatric surgery as a novel treatment for type 2 diabetes mellitus: a systematic review. Arch Surg 2011;146:744-50.

27. Lean ME, Leslie WS, Barnes AC, et al. Primary care-led weight management for remission of type 2 diabetes (DiRECT): an openlabel, cluster-randomised trial. Lancet 2018;391.

28. Jackness C, Karmally W, Febres G, et al. Very low-calorie diet mimics the early beneficial effect of Roux-en-Y gastric bypass on insulin sensitivity and $\beta$-cell Function in type 2 diabetic patients. Diabetes 2013;62:3027-32.

29. Lim EL, Hollingsworth KG, Aribisala BS, et al. Reversal of type 2 diabetes: normalisation of beta cell function in association with decreased pancreas and liver triacylglycerol. Diabetologia 2011;54:2506-14.

30. Jazet IM, Schaart G, Gastaldelli A, et al. Loss of $50 \%$ of excess weight using a very low energy diet improves insulin-stimulated glucose disposal and skeletal muscle insulin signalling in obese insulin-treated type 2 diabetic patients. Diabetologia 2008;51:309-19.

31. Steven S, Lim EL, Taylor R. Population response to information on reversibility of Type 2 diabetes. Diabet Med 2013;30:e135-e138.

32. Rider OJ, Francis JM, Ali MK, et al. Beneficial cardiovascular effects of bariatric surgical and dietary weight loss in obesity. J Am Coll Cardiol 2009;54:718-26

33. Rider OJ, Francis JM, Tyler D, et al. Effects of weight loss on myocardial energetics and diastolic function in obesity. Int $J$ Cardiovasc Imaging 2013;29:1043-50.

34. Utz W, Engeli S, Haufe S, et al. Moderate dietary weight loss reduces myocardial steatosis in obese and overweight women. Int J Cardiol 2013;167:905-9.

35. Hammer S, Snel M, Lamb HJ, et al. Prolonged caloric restriction in obese patients with type 2 diabetes mellitus decreases myocardial triglyceride content and improves myocardial function. J Am Coll Cardiol 2008;52:1006-12.

36. Jonker JT, Djaberi R, van Schinkel LD, et al. Very-low-calorie diet increases myocardial triglyceride content and decreases diastolic left ventricular function in type 2 diabetes with cardiac complications. Diabetes Care 2014;37:e1-e2.

37. Christensen P, Bliddal H, Riecke BF, et al. Comparison of a low-energy diet and a very low-energy diet in sedentary obese individuals: a pragmatic randomized controlled trial. Clin Obes 2011;1:31-40.

38. Bassuk SS, Manson JE. Epidemiological evidence for the role of physical activity in reducing risk of type 2 diabetes and cardiovascular disease. J Appl Physiol 2005;99:1193-204.

39. Jeon CY, Lokken RP, Hu FB, et al. Physical activity of moderate intensity and risk of type 2 diabetes: a systematic review. Diabetes Care 2007;30:744-52.

40. Boulé NG, Haddad E, Kenny GP, et al. Effects of exercise on glycemic control and body mass in type 2 diabetes mellitus: a metaanalysis of controlled clinical trials. JAMA 2001;286:1218-27.
41. Umpierre D, Ribeiro PA, Kramer CK, et al. Physical activity advice only or structured exercise training and association with $\mathrm{HbA} 1 \mathrm{c}$ levels in type 2 diabetes: a systematic review and meta-analysis. JAMA 2011;305:1790-9.

42. Hawley JA. Exercise as a therapeutic intervention for the prevention and treatment of insulin resistance. Diabetes Metab Res Rev 2004;20:383-93.

43. Telford RD. Low physical activity and obesity: causes of chronic disease or simply predictors?. Med Sci Sports Exerc 2007;39:1233-40.

44. Schuster I, Vinet A, Karpoff L, et al. Diastolic dysfunction and intraventricular dyssynchrony are restored by low intensity exercise training in obese men. Obesity 2012;20-134-40.

45. Sandri M, Kozarez I, Adams V, et al. Age-related effects of exercise training on diastolic function in heart failure with reduced ejection fraction: the Leipzig Exercise Intervention in Chronic Heart Failure and Aging (LEICA) Diastolic Dysfunction Study. Eur Heart J 2012;33:1758-68.

46. Brassard P, Legault S, Garneau C, et al. Normalization of diastolic dysfunction in type 2 diabetics after exercise training. Med Sci Sports Exerc 2007;39:1896-901.

47. Hordern MD, Coombes JS, Cooney LM, et al. Effects of exercise intervention on myocardial function in type 2 diabetes. Heart 2009;95:1343-9.

48. Smart N, Haluska B, Jeffriess L, et al. Exercise training in systolic and diastolic dysfunction: effects on cardiac function, functional capacity, and quality of life. Am Heart J 2007;153:530-6.

49. Cho NH, Shaw JE, Karuranga S, et al. IDF Diabetes Atlas: Global estimates of diabetes prevalence for 2017 and projections for 2045. Diabetes Res Clin Pract 2018;138:271-81.

50. Matthews DR, Hosker JP, Rudenski AS, et al. Homeostasis model assessment: insulin resistance and beta-cell function from fasting plasma glucose and insulin concentrations in man. Diabetologia 1985;28:412-9.

51. von Bibra H, Saha S, Hapfelmeier A, et al. Impact of the triglyceride/ high-density lipoprotein cholesterol ratio and the hypertriglyceremicwaist phenotype to predict the metabolic syndrome and insulin resistance. Horm Metab Res 2017;49:542-9.

52. Wasserman K. Principles of Exercise Testing and Interpretation: Including Pathophysiology and Clinical Applications: Lippincott Williams \& Wilkins, 2005.

53. Rider OJ, Lewandowski A, Nethononda R, et al. Gender-specific differences in left ventricular remodelling in obesity: insights from cardiovascular magnetic resonance imaging. Eur Heart $J$ 2013;34:292-9

54. NICE, N.C.C.f.C.C.a.t.C.f.C.P.a., The management of type 2 diabetes NICE clinical guideline. 2010;87

55. . Excellence, N.I.f.H.a.C., NICE guideline [NG28] Type 2 diabetes in adults: management. 2015, NICE

56. Carlo Agostoni RBC, Fairweather-Tait S, Heinonen M, et al. Scientific Opinion on the essential composition of total diet replacements for weight control. EFSA Journal 2015;13:52.

57. Singh A, Steadman CD, Khan JN, et al. Intertechnique agreement and interstudy reproducibility of strain and diastolic strain rate at 1.5 and 3 Tesla: a comparison of feature-tracking and tagging in patients with aortic stenosis. J Magn Reson Imaging 2015;41:1129-37.

58. Mahmod M, Bull S, Suttie JJ, et al. Myocardial steatosis and left ventricular contractile dysfunction in patients with severe aortic stenosis. Circ Cardiovasc Imaging 2013;6:808-16.

59. Htike ZZ, Yates T, Brady EM, et al. Rationale and design of the randomised controlled trial to assess the impact of liraglutide on cardiac function and structure in young adults with type 2 diabetes (the LYDIA study). Cardiovasc Diabetol 2016;15:102.

60. Scatteia A, Baritussio A, Bucciarelli-Ducci C. Strain imaging using cardiac magnetic resonance. Heart Fail Rev 2017;22:465-76.

61. Pedrizzetti G, Claus P, Kilner PJ, et al. Principles of cardiovascular magnetic resonance feature tracking and echocardiographic speckle tracking for informed clinical use. J Cardiovasc Magn Reson 2016;18:51.

62. Khan JN, Singh A, Nazir SA, et al. Comparison of cardiovascular magnetic resonance feature tracking and tagging for the assessment of left ventricular systolic strain in acute myocardial infarction. Eur J Radiol 2015;84:840-8.

63. St Pierre TG, House MJ, Bangma SJ, et al. Stereological Analysis of Liver Biopsy Histology Sections as a Reference Standard for Validating Non-Invasive Liver Fat Fraction Measurements by MRI. PLoS One 2016;11:e0160789.

64. Steadman CD, Jerosch-Herold M, Grundy B, et al. Determinants and functional significance of myocardial perfusion reserve in severe aortic stenosis. JACC Cardiovasc Imaging 2012;5:182-9. 
65. Wharton G, Steeds R, Allen J, et al. A minimum dataset for a standard adult transthoracic echocardiogram: a guideline protocol from the British Society of Echocardiography. Echo Res Pract 2015;2:G9-G24.

66. Almeida JG, Fontes-Carvalho R, Sampaio F, et al. Impact of the 2016 ASE/EACVI recommendations on the prevalence of diastolic dysfunction in the general population. Eur Heart J Cardiovasc Imaging 2018;19:380-6.

67. Nauta JF, Hummel YM, van der Meer P, et al. Correlation with invasive left ventricular filling pressures and prognostic relevance of the echocardiographic diastolic parameters used in the 2016 ESC heart failure guidelines and in the 2016 ASE/EACV recommendations: a systematic review in patients with heart failure with preserved ejection fraction. Eur J Heart Fail 2018;20:1303-11.

68. von Bibra H, Paulus WJ, St John Sutton M, et al. Quantification of diastolic dysfunction via the age dependence of diastolic function impact of insulin resistance with and without type 2 diabetes. Int $J$ Cardiol 2015;182:368-74.

69. Das $\mathrm{P}$, Rimington $\mathrm{H}$, Smeeton $\mathrm{N}$, et al. Determinants of symptoms and exercise capacity in aortic stenosis: a comparison of resting haemodynamics and valve compliance during dobutamine stress. Eur Heart J 2003;24:1254-63.

70. Yates T, Griffin S, Bodicoat DH, et al. PRomotion Of Physical activity through structured Education with differing Levels of ongoing Support for people at high risk of type 2 diabetes (PROPELS): study protocol for a randomized controlled trial. Trials 2015;16:289.

71. Besson H, Brage S, Jakes RW, et al. Estimating physical activity energy expenditure, sedentary time, and physical activity intensity by self-report in adults. Am J Clin Nutr 2010;91:106-14.

72. Paradisi G, Smith L, Burtner C, et al. Dual energy X-ray absorptiometry assessment of fat mass distribution and its association with the insulin resistance syndrome. Diabetes Care $1999 ; 22: 1310-7$
73. Bertin E, Marcus C, Ruiz JC, et al. Measurement of visceral adipose tissue by DXA combined with anthropometry in obese humans. Int $J$ Obes Relat Metab Disord 2000;24:263-70.

74. Paulus WJ, Tschöpe C, Sanderson JE, et al. How to diagnose diastolic heart failure: a consensus statement on the diagnosis of heart failure with normal left ventricular ejection fraction by the Heart Failure and Echocardiography Associations of the European Society of Cardiology. Eur Heart J 2007;28:2539-50.

75. Kadappu KK, Boyd A, Eshoo S, et al. Changes in left atrial volume in diabetes mellitus: more than diastolic dysfunction? Eur Heart $J$ Cardiovasc Imaging 2012;13:1016-23.

76. Rayner JJ, Banerjee R, Francis JM, et al. Normalization of visceral fat and complete reversal of cardiovascular remodeling accompany gastric bypass, not banding. J Am Coll Cardiol 2015;66:2569-70.

77. Sattar N, Petrie MC, Zinman B, et al. Novel diabetes drugs and the cardiovascular specialist. J Am Coll Cardiol 2017;69:2646-56.

78. James PA, Oparil S, Carter BL, et al. 2014 evidence-based guideline for the management of high blood pressure in adults: report from the panel members appointed to the Eighth Joint National Committee (JNC 8). JAMA 20142014;311:507-20.

79. Leung AA, Nerenberg K, Daskalopoulou SS, et al. Hypertension canada's 2016 canadian hypertension education program guidelines for blood pressure measurement, diagnosis, assessment of risk, prevention, and treatment of hypertension. Can J Cardiol 2016;32:569-88.

80. Urata $\mathrm{H}$, Tanabe $\mathrm{Y}$, Kiyonaga A, et al. Antihypertensive and volumedepleting effects of mild exercise on essential hypertension. Hypertension 1987;9:245-52.

81. Duncan JJ, Farr JE, Upton SJ, et al. The effects of aerobic exercise on plasma catecholamines and blood pressure in patients with mild essential hypertension. JAMA 1985;254:2609-13. 